\title{
Two-sided optimal stopping for Lévy processes
}

\author{
Ernesto Mordecki* Facundo Oliú Eguren ${ }^{\dagger}$
}

\begin{abstract}
Infinite horizon optimal stopping problems for a Lévy processes with a two-sided reward function are considered. A two-sided verification theorem is presented in terms of the overall supremum and the overall infimum of the process. A result to compute the angle of the value function at the optimal thresholds of the stopping region is given. To illustrate the results, the optimal stopping problem of a compound Poisson process with two-sided exponential jumps and a two-sided payoff function is solved. In this example, the smooth-pasting condition does not hold.
\end{abstract}

Keywords: optimal stopping; Lévy processes, two sided problems.

MSC2020 subject classifications: $60 \mathrm{G} 40$.

Submitted to ECP on December 13, 2019, final version accepted on January 27, 2021.

Supersedes arXiv: 1912.08171v1.

\section{Introduction}

The presence of the maximum in the solution of optimal stopping problems for Lévy processes is nowadays understood, as it was summarized in the monograph by Kyprianou [8]. After the work of Surya [15] (see also Mordecki and Mishura [12]) it became clear that one sided problems with arbitrary payoff functions could be solved with the help of an averaging function. More recently, some formulas appeared for two sided problems, where the infimum and the supremum took part in the solution. For instance, in Mordecki and Salminen [13], with the help of representation techniques, a formula for the value function through the sum two averaging functions (one for the maximum and another for the minimum) was obtained. Afterwards, a similar formula was obtained through verification techniques by Christensen et al. [6]. This second result involved the supremum of the two averaging functions.

The purpose of the present work is then to obtain a verification theorem for the optimal stopping problem of a Lévy process in the two sided case, through the sum of two averaging functions, and to provide a simple example that seems not possible to be solved with the existing techniques.

References on optimal stopping problems for Lévy processes with two sided solutions, to our knowledge, are few. Perpetual American strangle options are considered by Chang and Sheu [5], through the solution of a free boundary integro-differential problem with moving boundaries. In this case, the smooth pasting condition is a key ingredient, and a

\footnotetext{
*Centro de Matemática, Facultad de Ciencias, Universidad de la República, Uruguay. E-mail: mordecki@ cmat.edu.uy

${ }^{\dagger}$ Centro de Matemática, Facultad de Ciencias, Universidad de la República, Uruguay. E-mail: facundooliu@ gmail.com
} 
non vanishing gaussian part in the Lévy process is used to ensure this condition. These results follow previously obtained unpublished ones by Boyarchenko [3]. Similar type of techniques were applied in Buonaguidi and Muliere [4] to solve a statistical problem: the Bayesian sequential testing of two simple hypothesis. More recently, De Donno et al. [7] found disconnected continuation regions in American put options with negative discount rates in Lévy models.

The content of the rest of the paper is as follows. In section 2 we formulate the verification result for two-sided optimal stopping with the corresponding proof. In section 3 a result to compute the angle (i.e. the difference of the right and left derivatives) of the value function at a critical threshold is obtained. Section 4 contains an example: the optimal stopping problem of a compound Poission process with two-sided exponential jumps, (no gaussian component) and payoff function $g(x)=|x|$.

\section{A verification result for optimal stopping}

Let $X=\left\{X_{t}: t \geq 0\right\}$ be a Lévy process defined on a stochastic basis $(\Omega, \mathcal{F}, \mathbf{F}=$ $\left.\left(\mathcal{F}_{t}\right)_{t \geq 0}, \mathbf{P}_{x}\right)$ departing from $X_{0}=x$. The corresponding expectation is denoted by $\mathbf{E}_{x}$, and for short we denote $\mathbf{E}=\mathbf{E}_{0}$ and $\mathbf{P}=\mathbf{P}_{0}$. The Lévy-Khintchine formula characterizes the law of the process, stating, for $z \in i \mathbb{R}$, that $\mathbf{E} e^{z X_{t}}=e^{t \psi(z)}$ with

$$
\psi(z)=a z+\frac{\sigma^{2}}{2} z^{2}+\int_{\mathbb{R}}\left(e^{z y}-1-z h(y)\right) \Pi(d y),
$$

where $a \in \mathbb{R}, \sigma \geq 0$ and $\Pi(d y)$ is a non-negative measure (the jump measure) that satisfies $\int_{\mathbb{R}}\left(1 \wedge y^{2}\right) \Pi(d y)<\infty$. Here $h(y)=y \mathbf{1}_{\{|y|<1\}}$ is a truncation function. For general references on Lévy processes see Bertoin [2] or Kyprianou [8]. The set of stopping times is the set of random variables

$$
\mathcal{M}=\left\{\tau: \Omega \rightarrow[0, \infty] \text { such that }\{\tau \leq t\} \in \mathcal{F}_{t} \text { for all } t \geq 0\right\} .
$$

Observe that we allow the possibility $\tau=\infty$ as for several optimal stopping problems, the optimal stopping time is within this class. A key rôle in the solution of two-sided problems is played by the overall supremum and infimum of the process, defined respectively by

$$
M=\sup \left\{X_{t}: 0 \leq t \leq e_{r}\right\}, \quad I=\inf \left\{X_{t}: 0 \leq t \leq e_{r}\right\},
$$

where $e_{r}$ is an exponential random variable of parameter $r>0$, independent of $X$. Observe that as $r>0$ both random variables $M$ and $I$ are proper.

Given a non-negative continuous payoff function $g(x)$, a Lévy process $X$, and a discount factor $r>0$, the optimal stopping problem (OSP) consists in finding the value function $V(x)$ and the optimal stopping rule $\tau^{*}$ such that

$$
V(x)=\sup _{\tau \in \mathcal{M}} \mathbf{E}_{x}\left(e^{-r \tau} g\left(X_{\tau}\right)\right)=\mathbf{E}_{x}\left(e^{-r \tau^{*}} g\left(X_{\tau^{*}}\right)\right) .
$$

We assume that the payoff received in the set $\{\omega: \tau(\omega)=\infty\}$ is zero, in fact, we identify

$$
e^{-r \tau} g\left(X_{\tau}\right)=e^{-r \tau} g\left(X_{\tau}\right) \mathbf{1}_{\{\tau<\infty\}} .
$$

In the present paper we are interested in problems with two-sided solutions, i.e. such that the optimal stopping rule is of the form

$$
\tau^{*}=\inf \left\{t \geq 0: X_{t} \notin\left(-x_{1}, x_{2}\right)\right\},
$$

for some critical thresholds $-x_{1}<0<x_{2}$. As the process is space-invariant, the thresholds are chosen negative and positive for simple convenience of notation. Observe that, having into account the asymptotic behavior of a Lévy process (see Thm. VI.12 in Bertoin [2]) the stopping time in (2.2) satisfies $\mathbf{P}\left(\tau^{*}<\infty\right)=1$. 
Theorem 2.1. Consider a Lévy process $X$, a discount rate $r>0$, and a continuous reward function $g: \mathbb{R} \rightarrow[0, \infty)$. Assume that there exist two points $-x_{1}<0<x_{2}$ and two continuous monotonous functions: $Q_{1}$ non-increasing with $Q_{1}(x)=0$ for $-x_{1} \leq x ; Q_{2}$ non-decreasing with $Q_{2}(x)=0$ for $x \leq x_{2}$ (named averaging functions); and such that

$$
g(x)=\mathbf{E}_{x} Q_{1}(I)+\mathbf{E}_{x} Q_{2}(M), \text { for all } x \notin\left(-x_{1}, x_{2}\right) .
$$

Define the function

$$
V(x)=\mathbf{E}_{x}\left(Q_{1}(I)+Q_{2}(M)\right), \text { for all } x \in \mathbb{R} .
$$

Then, if the condition

$$
V(x) \geq g(x),
$$

holds for all $x \in\left[-x_{1}, x_{2}\right]$, the OSP (2.1) has value function $V(x)$ in (2.4), and (2.2) is an optimal stopping time for the problem.

As usual in optimal stopping, in order to prove Theorem 2.1, we verify two statements:

$$
\begin{aligned}
& V(x) \geq \mathbf{E}\left(e^{-r \tau} g\left(X_{\tau}\right)\right), \quad \forall \tau \in \mathcal{M}, \\
& V(x)=\mathbf{E}\left(e^{-r \tau^{*}} g\left(X_{\tau^{*}}\right)\right) .
\end{aligned}
$$

The proof of these two facts are stated in two corresponding lemmas. Their proofs follow, with the necessary modifications, from the respective proofs in Mordecki and Mishura [12]. For a definition of $r$-excessive function see Christensen et al. [6].

Lemma 2.2. For a Lévy process and $r>0$, consider two non-negative continuous functions: $f(x)$ non-decreasing; $g(x)$ non-increasing. Then:

(a) The function

$$
h(x)=\mathbf{E}_{x}(f(M)+g(I)) \quad(x \in \mathbb{R})
$$

is $r$-excessive, and, in consequence,

(b) the process $\left\{e^{-r t} h\left(X_{t}\right): t \geq 0\right\}$ is a supermartingale.

Proof. The fact that (b) follows from (a) is standard. In fact, if $h$ is $r$-excessive

$$
\begin{aligned}
e^{-r(t+h)} \mathbf{E}_{x}\left(h\left(X_{t+h}\right) \mid \mathcal{F}_{t}\right) & =e^{-r(t+h)} \mathbf{E}_{x}\left(h\left(X_{h} \circ \theta_{t}\right) \mid \mathcal{F}_{t}\right) \\
& =e^{-r t} e^{-r h} \mathbf{E}_{X_{t}} h\left(X_{h}\right) \leq e^{-r t} h\left(X_{t}\right),
\end{aligned}
$$

where $\theta_{t}$ is the shift operator, giving the super-martingale property. To prove (a) we know, from Lemma 2.2 in Christensen et al. [6], that the following two functions are excessive:

$$
\begin{aligned}
& u(x):=\mathbf{E}_{x} \sup _{0 \leq t \leq e_{r}} f\left(X_{t}\right)=\mathbf{E}_{x} f\left(\sup _{0 \leq t \leq e_{r}} X_{t}\right)=\mathbf{E}_{x} f(M), \\
& v(x):=\mathbf{E}_{x} \sup _{0 \leq t \leq e_{r}} g\left(X_{t}\right)=\mathbf{E}_{x} g\left(\inf _{0 \leq t \leq e_{r}} X_{t}\right)=\mathbf{E}_{x} g(I) .
\end{aligned}
$$

The proof concludes as $h=u+v$, and excessivity is preserved by summation. 
Remark 2.3. When comparing the representations of payoffs for two-sided problems in Theorem 2.7 in Christensen et al. [6] and Proposition 4.4. in Mordecki and Salminen [13], it should be noticed that in the first case, the excessive function is $\mathbf{E}_{x}(f(M) \vee g(I))$, and in the second, $\mathbf{E}_{x}(f(M)+g(I))$. These two constructions give different representations of the payoff of the problem. In the present work we use the second one.

Lemma 2.4. Consider a Lévy process $X$, a discount rate $r>0$, and functions $Q_{1}, Q_{2}$ and $g$ such that (2.3) and (2.5) hold, for $V$ defined by (2.4). Then (2.7) holds.

Proof. Denote $S=\mathbb{R} \backslash\left(-x_{1}, x_{2}\right)$. As $X_{\tau^{*}} \in S$ and $V=g$ on $S$, we have

$$
\mathbf{E}_{x}\left(e^{-r \tau^{*}} g\left(X_{\tau^{*}}\right)\right)=\mathbf{E}_{x}\left(e^{-r \tau^{*}} V\left(X_{\tau^{*}}\right)\right) .
$$

On the other side,

$$
V(x)=\mathbf{E}_{x}\left(Q_{1}(I)+Q_{2}(M)\right)=\mathbf{E}_{x}\left(\sup _{0 \leq t \leq e_{r}} Q_{1}\left(X_{t}\right)+\sup _{0 \leq t \leq e_{r}} Q_{2}\left(X_{t}\right)\right) .
$$

Observe that if $e_{r}<\tau^{*}$ we have $M<x_{2}$ and $I>-x_{1}$, and then

$$
\sup _{0 \leq t \leq e_{r}} Q_{1}\left(X_{t}\right)+\sup _{0 \leq t \leq e_{r}} Q_{2}\left(X_{t}\right)=0
$$

because $Q_{1}(x)=Q_{2}(x)=0$ in $\left[-x_{1}, x_{2}\right]$. So, denoting

$$
\begin{aligned}
& V_{1}(x):=\mathbf{E}_{x}\left(\sup _{\tau^{*} \leq t \leq e_{r}} Q_{1}\left(X_{t}\right)\right)=\mathbf{E}_{x} Q_{1}(I), \\
& V_{2}(x):=\mathbf{E}_{x}\left(\sup _{\tau^{*} \leq t \leq e_{r}} Q_{2}\left(X_{t}\right)\right)=\mathbf{E}_{x} Q_{2}(M) .
\end{aligned}
$$

we have

$$
V(x)=V_{1}(x)+V_{2}(x) .
$$

Consider now $\tilde{X}=\left\{\tilde{X}_{s}=X_{\tau^{*}+s}-X_{\tau^{*}}: s \geq 0\right\}$ that, by the strong Markov property, is independent of $\mathcal{F}_{\tau^{*}}$ and has the same distribution as $X$, and denote by $\tilde{\mathbf{E}}_{x}$ the expectation w.r.t. $\tilde{X}$. Based on these considerations, we have

$$
\begin{aligned}
V_{1}(x) & =\mathbf{E}_{x}\left(\sup _{\tau^{*} \leq t \leq e_{r}} Q_{1}\left(X_{t}\right)\right)=\mathbf{E}_{x}\left(\int_{\tau^{*}}^{\infty} \sup _{\tau^{*} \leq t \leq u} Q_{1}\left(X_{t}\right) r e^{-r u} d u\right) \\
& =\mathbf{E}_{x}\left(e^{-r \tau^{*}} \int_{0}^{\infty} \sup _{\tau^{*} \leq t \leq \tau^{*}+v} Q_{1}\left(X_{t}\right) r e^{-r v} d v\right) \\
& =\mathbf{E}_{x}\left(e^{-r \tau^{*}} \int_{0}^{\infty} \sup _{\tau^{*} \leq t \leq \tau^{*}+v} Q_{1}\left(X_{\tau^{*}}+X_{t}-X_{\tau^{*}}\right) r e^{-r v} d v\right) \\
& =\mathbf{E}_{x}\left(e^{-r \tau^{*}} \int_{0}^{\infty} \sup _{0 \leq s \leq v} Q_{1}\left(X_{\tau^{*}}+X_{\tau^{*}+s}-X_{\tau^{*}}\right) r e^{-r v} d v\right) \\
& =\mathbf{E}_{x}\left(e^{-r \tau^{*}} \int_{0}^{\infty} \sup _{0 \leq s \leq v} Q_{1}\left(X_{\tau^{*}}+\tilde{X}_{s}\right) r e^{-r v} d v\right) \\
& =\mathbf{E}_{x}\left(e^{-r \tau^{*}} \tilde{\mathbf{E}}_{X_{\tau^{*}}}\left[\int_{0}^{\infty} \sup _{0 \leq s \leq v} Q_{1}\left(\tilde{X}_{s}\right) r e^{-r v} d v\right]\right) \\
& =\mathbf{E}_{x}\left(e^{-r \tau^{*}} \tilde{\mathbf{E}}_{X_{\tau^{*}}}\left[\sup _{0 \leq s \leq e_{r}} Q_{1}\left(\tilde{X}_{s}\right)\right]\right)=\mathbf{E}_{x}\left(e^{-r \tau^{*}} V_{1}\left(X_{\tau^{*}}\right)\right),
\end{aligned}
$$

where we change variables according to $v=u-\tau^{*}$ to pass from (2.11) to (2.12), and denote $s=t-\tau^{*}$ to pass from (2.13) to (2.14). The same relation holds with $V_{2}$ and $Q_{2}$. Summing up these two relations, and in view of (2.8), we conclude the proof of the Lemma. 
Proof of Theorem 2.1. The proof now follows, as, according to Lemma 2.2 the nonnegative process $\left\{e^{-r t} V\left(X_{t}\right): t \geq 0\right\}$ is a supermartingale, giving

$$
V(x) \geq \mathbf{E}\left(e^{-r \tau} V\left(X_{\tau}\right)\right) \geq \mathbf{E}\left(e^{-r \tau} g\left(X_{\tau}\right)\right),
$$

by Doob's optional sampling Theorem first and the application of condition (2.5) second. This establishes (2.6). On its turn, Lemma 2.4 gives equality (2.7), concluding the proof.

\section{On the smooth pasting condition}

Smooth pasting results for general Lévy processes were obtained for put American perpetual options for Lévy processes in Alili and Kyprianou [1], and afterwards generalized to put-type (bounded) payoffs by Surya [15]. The following result gives some natural necessary conditions for smooth pasting, that depend on the exponential moments of the process and the behaviour of the averaging function. They can be applied both to one-sided problems (as the ones considered in Mordecki and Mishura [12]) and to the two sided problems considered in the present paper.

Theorem 3.1. Consider a Lévy process $X$, a discount rate $r>0$ and a continuous reward function $g: \mathbb{R} \rightarrow[0, \infty)$. Assume that there exist a point $x_{0}$ and a continuous non-decreasing averaging function $Q$ with $Q(x)=0$ for $x \leq x_{0}$, such that

$$
g(x)=\mathbf{E}_{x} Q(M), \text { for all } x \geq x_{0} .
$$

Assume that $Q \in C^{2}\left[x_{0}, \infty\right)$, and satisfies

$$
\left|Q^{\prime \prime}(x)\right| \leq A e^{\alpha x}, \forall x \geq x_{0},
$$

for some $A>0$ and some $\alpha>0$. Regarding the process, assume that

$$
\mathbf{E} e^{\alpha X_{1}}<e^{r} .
$$

Then, the candidate to value function of the OSP (2.1)

$$
V(x)=\mathbf{E}_{x} Q(M), \text { for all } x \in \mathbb{R}
$$

satisfies

$$
V^{\prime}\left(x_{0}+\right)-V^{\prime}\left(x_{0}-\right)=Q^{\prime}\left(x_{0}+\right) \mathbf{P}(M=0),
$$

and

$$
V^{\prime}(x+)-V^{\prime}(x-)=0, \quad \text { for } x>x_{0} .
$$

Proof. We first prove (3.2). Denoting by $F_{M}(y)(y \geq 0)$ the distribution function of $M$, we have

$$
\begin{aligned}
V^{\prime}\left(x_{0}+\right)-V^{\prime}\left(x_{0}-\right) \\
=\lim _{h \downarrow 0} \frac{1}{h} \mathbf{E}\left[Q\left(x_{0}+h+M\right)+Q\left(x_{0}-h-M\right)-2 Q\left(x_{0}+M\right)\right] \\
=\lim _{h \downarrow 0} \frac{1}{h} \int_{[0, \infty)}\left[Q\left(x_{0}+h+y\right)+Q\left(x_{0}-h-y\right)-2 Q\left(x_{0}+y\right)\right] d F_{M}(y) \\
=\lim _{h \downarrow 0} \frac{1}{h}\left\{\left[Q\left(x_{0}+h\right)+Q\left(x_{0}-h\right)-2 Q\left(x_{0}\right)\right] \mathbf{P}(M=0)\right. \\
\quad+\int_{(0, h)}\left[Q\left(x_{0}+h+y\right)+Q\left(x_{0}-h+y\right)-2 Q\left(x_{0}+y\right)\right] d F_{M}(y) \\
\left.\quad+\int_{(h, \infty)}\left[Q\left(x_{0}+h+y\right)+Q\left(x_{0}-h+y\right)-2 Q\left(x_{0}+y\right)\right] d F_{M}(y)\right\} .
\end{aligned}
$$


To compute the limit in (3.4), as $Q(x)=0$ for $x \leq x_{0}$, we have

$$
\lim _{h \downarrow 0} \frac{1}{h}\left[Q\left(x_{0}+h\right)+Q\left(x_{0}-h\right)-2 Q\left(x_{0}\right)\right] \mathbf{P}(M=0)=Q^{\prime}\left(x_{0}+\right) \mathbf{P}(M=0) .
$$

Concerning (3.5), we have

$$
\begin{array}{r}
\lim _{h \downarrow 0} \frac{1}{h}\left|\int_{(0, h)}\left[Q\left(x_{0}+h+y\right)+Q\left(x_{0}-h+y\right)-2 Q\left(x_{0}+y\right)\right] d F_{M}(y)\right| \\
\leq 4 \lim _{h \downarrow 0} \frac{Q\left(x_{0}+2 h\right)}{h} \mathbf{P}(0<M \leq h)=8 Q\left(x_{0}\right)^{\prime} \lim _{h \downarrow 0} \mathbf{P}(0<M \leq h)=0 .
\end{array}
$$

To consider the term in (3.6), denote $x=x_{0}+y \geq x_{0}+h$,

$$
\begin{array}{r}
|Q(x+h)+Q(x-h)-2 Q(x)|=\left|\int_{x}^{x+h} d u \int_{u-h}^{u} Q^{\prime \prime}(v) d v\right| \\
\leq A \int_{x}^{x+h} d u \int_{u-h}^{u} e^{\alpha v} d v=\frac{A}{\alpha^{2}}\left(e^{\alpha(x+h)}+e^{\alpha(x-h)}-2 e^{\alpha x}\right) .
\end{array}
$$

We now apply Lemma 1 in Mordecki [10] to the Lévy process $\alpha X$, to obtain that condition (3.1) implies that $\mathbf{E} e^{\alpha M}<\infty$. In consequence, we have

$$
\begin{gathered}
\left|\int_{(h, \infty)}\left[Q\left(x_{0}+h+y\right)+Q\left(x_{0}-h+y\right)-2 Q\left(x_{0}+y\right)\right] d F_{M}(y)\right| \\
\leq \frac{A}{\alpha^{2}} \mathbf{E}\left(e^{\alpha\left(x_{0}+h+M\right)}+e^{\alpha\left(x_{0}-h+M\right)}-2 e^{\alpha\left(x_{0}+M\right)}\right) \\
\leq \frac{A}{\alpha^{2}}\left(e^{\alpha\left(x_{0}+h\right)}+e^{\alpha\left(x_{0}-h\right)}-2 e^{\alpha x_{0}}\right) \mathbf{E} e^{\alpha M} .
\end{gathered}
$$

In conclussion

$$
\lim _{h \downarrow 0} \frac{1}{h} \int_{(h, \infty)}\left[Q\left(x_{0}+h+y\right)+Q\left(x_{0}-h+y\right)-2 Q\left(x_{0}+y\right)\right] d F_{M}(y)=0,
$$

concluding the proof of (3.2). To verify (3.3) the same computations apply with $x$ instead of $x_{0}$. The difference is that, in the term (3.4), we have now

$$
\lim _{h \downarrow 0} \frac{1}{h}[Q(x+h)+Q(x-h)-2 Q(x)] \mathbf{P}(M=0)=\left(Q^{\prime}(x+)-Q^{\prime}(x-)\right) \mathbf{P}(M=0)=0 .
$$

This concludes the proof of the Theorem.

\section{An application}

To illustrate our results, we consider a compound Poisson process $X=\left\{X_{t}: t \geq 0\right\}$ with double-sided exponential jumps, given by

$$
X_{t}=x-\sum_{i=1}^{N_{t}^{(1)}} Y_{i}^{(1)}+\sum_{i=1}^{N_{t}^{(2)}} Y_{i}^{(2)}
$$

where $N^{(1)}=\left\{N_{t}^{(1)}: t \geq 0\right\}$ and $N^{(2)}=\left\{N_{t}^{(2)}: t \geq 0\right\}$ are two Poisson processes with respective positive intensities $\lambda_{1}, \lambda_{2} ; Y^{(1)}=\left\{Y_{i}^{(1)}: i \geq 1\right\}$ and $Y^{(2)}=\left\{Y_{i}^{(2)}: i \geq 1\right\}$ are two sequences of independent exponentially distributed random variables with respective positive parameters $\alpha_{1}, \alpha_{2}$. The four processes $N^{(1)}, N^{(2)}, Y^{(1)}, Y^{(2)}$, are independent. We consider then the OSP (2.1) for the function $g(x)=|x|$ and the Lévy process $X$. 


\subsection{Wiener-Hopf factorization}

The characteristic exponent of $X$ is given by

$$
\psi(z)=-\lambda_{1} \frac{z}{\alpha_{1}+z}+\lambda_{2} \frac{z}{\alpha_{2}-z} .
$$

The equation $\psi(z)=r$ has two roots

$$
-r_{1}=\frac{\alpha_{1}\left(r+\lambda_{1}\right)}{r+\lambda_{1}+\lambda_{2}}, \quad r_{2}=\frac{\alpha_{2}\left(r+\lambda_{2}\right)}{r+\lambda_{1}+\lambda_{2}},
$$

that satisfy

$$
-\alpha_{1}<-r_{1}<0<r_{2}<\alpha_{2} .
$$

We apply the Wiener-Hopf factorization to determine the law of $M$ and $I$. We directly obtain the factors:

$$
\begin{aligned}
\frac{r}{r-\psi(z)} & =\frac{r\left(z-\alpha_{2}\right)\left(z-\alpha_{1}\right)}{(r+\lambda+\mu)\left(z-r_{2}\right)\left(z-r_{1}\right)}=\frac{r_{1} r_{2}\left(\alpha_{1}+z\right)\left(\alpha_{2}-z\right)}{\alpha_{1} \alpha_{2}\left(r_{1}+z\right)\left(r_{2}-z\right)} \\
& =\left(\frac{r_{1}}{\alpha_{1}}+\frac{\alpha_{1}-r_{1}}{\alpha_{1}} \frac{r_{1}}{r_{1}+z}\right)\left(\frac{r_{2}}{\alpha_{2}}+\frac{\alpha_{2}-r_{2}}{\alpha_{2}} \frac{r_{2}}{r_{2}-z}\right)
\end{aligned}
$$

In conclusion, due to the uniqueness of the factorization (see Thm. 5(ii) Ch. VI of Bertoin [2]), we obtain

$$
\mathbf{E} e^{z I}=\frac{r_{1}}{\alpha_{1}}+\frac{\alpha_{1}-r_{1}}{\alpha_{1}} \frac{r_{1}}{r_{1}+z}
$$

and

$$
\mathbf{E} e^{z M}=\frac{r_{2}}{\alpha_{2}}+\frac{\alpha_{2}-r_{2}}{\alpha_{2}} \frac{r_{2}}{r_{2}-z} .
$$

This means that the random variables $M$ and $-I$ have defective exponential distributions with parameters $r_{2}$ and $r_{1}$, and atoms at zero of respective size $r_{2} / \alpha_{2}$ and $r_{1} / \alpha_{1}$. With a slight abuse of notation, we denote these respective densities

$$
\begin{gathered}
f_{I}(x)=\frac{r_{1}}{\alpha_{1}} \delta_{0}(x)+\frac{\alpha_{1}-r_{1}}{\alpha_{1}} r_{1} e^{r_{1} x}, \quad x \leq 0, \\
f_{M}(x)=\frac{r_{2}}{\alpha_{2}} \delta_{0}(x)+\frac{\alpha_{2}-r_{2}}{\alpha_{2}} r_{2} e^{-r_{2} x}, \quad x \geq 0,
\end{gathered}
$$

where $\delta_{0}(x) d x$ denotes the Dirac mass measure at $x=0$. In order to introduce our result, we also need the following notations.

$$
\begin{array}{ll}
E_{1}=-\mathbf{E} I=\frac{1}{r_{1}}-\frac{1}{\alpha_{1}}>0, & E_{2}=\mathbf{E} M=\frac{1}{r_{2}}-\frac{1}{\alpha_{2}}>0, \\
F_{1}=\left(\mathbf{E} e^{r_{2} I}\right)^{-1}=\frac{\alpha_{1}}{r_{1}} \frac{r_{1}+r_{2}}{\alpha_{1}+r_{2}}>1, & F_{2}=\left(\mathbf{E} e^{-r_{1} M}\right)^{-1}=\frac{\alpha_{2}}{r_{2}} \frac{r_{1}+r_{2}}{r_{1}+\alpha_{2}}>1, \\
G_{1}=F_{1}-1=\frac{r_{2}\left(\alpha_{1}-r_{1}\right)}{r_{1}\left(\alpha_{1}+r_{2}\right)}>0, & G_{2}=F_{2}-1=\frac{r_{1}\left(\alpha_{2}-r_{2}\right)}{r_{2}\left(r_{1}+\alpha_{2}\right)}>0 .
\end{array}
$$

Theorem 4.1. Consider the Lévy process $X$ in (4.1), the payoff function $g(x)=|x|$, and $r>0$. Denote

$$
\begin{aligned}
& x_{1}=\frac{E_{1}\left(1-e^{-\left(r_{1}+r_{2}\right) u}\right)+F_{1} u e^{-r_{2} u}}{1+G_{1} e^{-\left(r_{1}+r_{2}\right) u}+F_{1} e^{-r_{2} u}}, \\
& x_{2}=\frac{E_{2}\left(1-e^{-\left(r_{1}+r_{2}\right) u}\right)+F_{2} u e^{-r_{1} u}}{1+G_{2} e^{-\left(r_{1}+r_{2}\right) u}+F_{2} e^{-r_{1} u}},
\end{aligned}
$$


where $u$ is the unique root of the equation

$$
u=\frac{E_{1}+E_{2}+\left(E_{1} G_{2}+E_{2} G_{1}\right) e^{-\left(r_{1}+r_{2}\right) u}+E_{1} F_{2} e^{-r_{1} u}+E_{2} F_{1} e^{-r_{2} u}}{1-G_{1} G_{2} e^{-\left(r_{1}+r_{2}\right) u}} .
$$

Denote

$$
D_{1}=\frac{x_{1}-x_{2} e^{-r_{2}\left(x_{1}+x_{2}\right)}}{1-e^{-\left(r_{1}+r_{2}\right)\left(x_{1}+x_{2}\right)}}, \quad D_{2}=\frac{x_{2}-x_{1} e^{-r_{1}\left(x_{1}+x_{2}\right)}}{1-e^{-\left(r_{1}+r_{2}\right)\left(x_{1}+x_{2}\right)}} .
$$

Then, the value function

$$
V(x)= \begin{cases}-x, & \text { for } x<-x_{1} \\ D_{1} e^{-r_{1}\left(x+x_{1}\right)}+D_{2} e^{r_{2}\left(x-x_{2}\right)}, & \text { for }-x_{1} \leq x \leq x_{2}, \\ x, & \text { for } x_{2}<x\end{cases}
$$

and stopping time defined in (2.2) conform the solution of the OSP (2.1).

Remark 4.2. An application of Theorem 3.1 (or more directly in this case, the computation of the corresponding derivatives in formula (4.9)) shows that the smooth pasting condition does not hold in any of the thresholds of the problem: the averaging functions have non-vanishing derivatives at the roots, and both the maximum and the infimum have atoms at the origin.

Proof. First observe that the r.h.s of (4.7) is decreasing, as the numerator decreases and the denominator increases with $u$ (all coefficients are positive). It decreases from

$$
\frac{2\left(E_{1} F_{2}+E_{2} F_{1}\right)}{1-G_{1} G_{2}}>0,
$$

when $u=0$ to $E_{1}+E_{2}$, as $u \rightarrow \infty$. Then, equation (4.7) has only one positive root. Here it was used that $G_{1} G_{2}<1$, fact that follows directly from the definitions (4.4). We need a technical result.

Lemma 4.3. Both equations (4.7) and

$$
u=\frac{E_{1}\left(1-e^{-\left(r_{1}+r_{2}\right) u}\right)+F_{1} u e^{-r_{2} u}}{1+G_{1} e^{-\left(r_{1}+r_{2}\right) u}+F_{1} e^{-r_{2} u}}+\frac{E_{2}\left(1-e^{-\left(r_{1}+r_{2}\right) u}\right)+F_{2} u e^{-r_{1} u}}{1+G_{2} e^{-\left(r_{1}+r_{2}\right) u}+F_{2} e^{-r_{1} u}},
$$

have a unique common positive root.

Proof of the Lemma. Denoting for short $\delta=r_{1}+r_{2}$, equation (4.10) reads

$$
u=\frac{E_{1}\left(1-e^{-\delta u}\right)+F_{1} u e^{-r_{2} u}}{1+G_{1} e^{-\delta u}+F_{1} e^{-r_{2} u}}+\frac{E_{2}\left(1-e^{-\delta u}\right)+F_{2} u e^{-r_{1} u}}{1+G_{2} e^{-\delta u}+F_{2} e^{-r_{1} u}} .
$$

Multiplying by the denominators, we obtain

$$
\begin{aligned}
u\left(1+G_{1} e^{-\delta u}+\right. & \left.F_{1} e^{-r_{2} u}\right)\left(1+G_{2} e^{-\delta u}+F_{2} e^{-r_{1} u}\right) \\
=\left(E_{1}\left(1-e^{-\delta u}\right)\right. & \left.+F_{1} u e^{-r_{2} u}\right)\left(1+G_{2} e^{-\delta u}+F_{2} e^{-r_{1} u}\right) \\
& +\left(E_{2}\left(1-e^{-\delta u}\right)+F_{2} u e^{-r_{1} u}\right)\left(1+G_{1} e^{-\delta u}+F_{1} e^{-r_{2} u}\right) .
\end{aligned}
$$

We first take on the l.h.s. the terms with the factor $u$. Afer computations in the l.h.s., many terms simplify, and in the r.h.s we take a common factor. The result of this computations is the equation

$$
\frac{u}{1-e^{-\delta u}}=\frac{E_{1}+E_{2}+\left(E_{1} G_{2}+E_{2} G_{1}\right) e^{-\delta u}+E_{2} F_{1} e^{-r_{2} u}+E_{1} F_{2} e^{-r_{1} u}}{1-\left(1+G_{1} G_{2}\right) e^{-\delta u}+G_{1} G_{2} e^{-2 \delta u}} .
$$


The denominator factorizes

$$
\begin{aligned}
1-\left(1+G_{1} G_{2}\right) e^{-\delta u}+ & G_{1} G_{2} e^{-2 \delta u}=1-e^{-\delta u}-G_{1} G_{2} e^{-\delta u}+G_{1} G_{2} e^{-2 \delta u} \\
& =1-e^{-\delta u}-G_{1} G_{2} e^{-\delta u}\left(1-e^{-\delta u}\right)=\left(1-e^{-\delta u}\right)\left(1-G_{1} G_{2} e^{-\delta u}\right)
\end{aligned}
$$

and equation (4.11) simplifies to (4.7), concluding the proof of the lemma.

We continue with the proof of Theorem 4.1. In view of the previous lemma and definitions (4.5) and (4.6), we obtain that $u=x_{1}+x_{2}$. In order to apply Theorem 2.1, introduce the functions

$$
\begin{aligned}
& Q_{1}(x)= \begin{cases}-x-E_{1}-F_{1} D_{2} e^{r_{2}\left(x-x_{2}\right)}, & \text { for } x \leq-x_{1}, \\
0, & \text { for } x>-x_{1},\end{cases} \\
& Q_{2}(x)= \begin{cases}0, & \text { for } x \leq x_{2}, \\
x-E_{2}-F_{2} D_{1} e^{-r_{1}\left(x+x_{1}\right)}, & \text { for } x \geq x_{2},\end{cases}
\end{aligned}
$$

We prove that these two functions are monotonous, non-increasing and non-decreasing respectively, and continuous. The equation:

$$
x_{1}=\frac{E_{1}\left(1-e^{-\left(r_{1}+r_{2}\right) u}\right)+F_{1} u e^{-r_{2} u}}{1+G_{1} e^{-\left(r_{1}+r_{2}\right) u}+F_{1} e^{-r_{2} u}},
$$

is equivalent to

$$
x_{1}=E_{1}+F_{1} \frac{x_{2}-x_{1} e^{-r_{1} u}}{1-e^{-\left(r_{1}+r_{2}\right) u}} e^{-r_{2} u}=E_{1}+F_{1} D_{2} e^{-r_{2}\left(x_{1}+x_{2}\right)} .
$$

We proceed to check the equivalence between (4.13) and (4.14) by using the definition of $G_{1}$ (4.4) and the equality $u=x_{1}+x_{2}$. Multiplying by the denominator in (4.13) we obtain

$$
x_{1}\left(1+F_{1} e^{-\left(r_{1}+r_{2}\right) u}-e^{-\left(r_{1}+r_{2}\right) u}+F_{1} e^{-r_{2} u}\right)=E_{1}\left(1-e^{-\left(r_{1}+r_{2}\right) u}\right)+F_{1} e^{-r_{2} u} u,
$$

that, after substitution of $u$ by $x_{1}+x_{2}$ and rearrengments gives,

$$
x_{1}\left(1-e^{-\left(r_{1}+r_{2}\right) u}\right)=E_{1}\left(1-e^{-\left(r_{1}+r_{2}\right) u}\right)-x_{1} F_{1} e^{-\left(r_{1}+r_{2}\right) u}+x_{2} F_{1} e^{-r_{2} u},
$$

that, dividing by the the $\left(1-e^{-\left(r_{1}+r_{2}\right) u}\right)$ gives the first equality in (4.14). The second one derives from the definition of $D_{2}$ (4.8), concluding the equivalence between (4.13) and (4.14).

Observe now that the statement (4.14) is equivalent to $Q_{1}\left(-x_{1}\right)=0$, giving the continuity of $Q_{1}$, that is clearly positive and non-increasing. Identical arguments apply to $Q_{2}$.

To check equality (2.3), consider first $x \geq-x_{1}$ and compute

$$
\begin{aligned}
& \mathbf{E}_{x} Q_{1}(I)=\int_{(-\infty, 0]} Q_{1}(x+y) f_{I}(y) d y \\
& =\int_{-\infty}^{x} Q_{1}(z) f_{I}(z-x) d z=\int_{-\infty}^{-x_{1}} Q_{1}(z) f_{I}(z-x) d z \\
& \quad=\frac{\alpha_{1}-r_{1}}{\alpha_{1}} e^{-r_{1}\left(x+x_{1}\right)}\left[x_{1}+\frac{1}{\alpha_{1}}-\frac{\alpha_{1}}{\alpha_{1}+r_{2}} D_{2} e^{-r_{2}\left(x_{1}+x_{2}\right)}\right],
\end{aligned}
$$


where we used the definition of $E_{1}$ in (4.2) and $F_{1}$ in (4.3). Observing that $D_{1}$ and $D_{2}$ are the solutions of the linear system of equations

$$
\left\{\begin{array}{l}
D_{1}+D_{2} e^{-r_{2}\left(x_{1}+x_{2}\right)}=x_{1}, \\
D_{1} e^{-r_{1}\left(x_{1}+x_{2}\right)}+D_{2}=x_{2},
\end{array}\right.
$$

we obtain

$$
\begin{aligned}
\mathbf{E}_{x} Q_{1}(I)=\frac{\alpha_{1}-r_{1}}{\alpha_{1}} e^{-r_{1}\left(x+x_{1}\right)}\left[x_{1}+\frac{1}{\alpha_{1}}-\frac{\alpha_{1}}{\alpha_{1}+r_{2}}\left(x_{1}-D_{1}\right)\right] \\
=\frac{\alpha_{1}-r_{1}}{\alpha_{1}} e^{-r_{1}\left(x+x_{1}\right)}\left[\frac{r_{2}}{\alpha_{1}+r_{2}} x_{1}+\frac{1}{\alpha_{1}}+\frac{\alpha_{1}}{\alpha_{1}+r_{2}} D_{1}\right] .
\end{aligned}
$$

Regarding the maximum, now for $x>x_{2}$,

$$
\begin{aligned}
& \mathbf{E}_{x} Q_{2}(M)=\frac{r_{2}}{\alpha_{2}} Q_{2}(x)+e^{r_{2} x} \int_{x}^{\infty} Q_{2}(z) f_{M}(z) d z \\
&=\frac{r_{2}}{\alpha_{2}}\left[x-E_{2}-F_{2} D_{1} e^{-r_{1}\left(x+x_{1}\right)}\right]+\frac{\alpha_{2}-r_{2}}{\alpha_{2}}\left[x+\frac{1}{\alpha_{2}}-\frac{\alpha_{2}}{r_{1}+\alpha_{2}} D_{1} e^{-r_{1}\left(x+x_{1}\right)}\right] \\
&=x-\frac{r_{2}}{\alpha_{2}} F_{2} D_{1} e^{-r_{1}\left(x+x_{1}\right)}-\frac{\alpha_{2}-r_{2}}{r_{1}+\alpha_{2}} D_{1} e^{-r_{1}\left(x+x_{1}\right)} \\
&=x-D_{1} e^{-r_{1}\left(x+x_{1}\right)} .
\end{aligned}
$$

Summing up,

$$
\begin{aligned}
& \mathbf{E}_{x} Q_{1}(I)+\mathbf{E}_{x} Q_{2}(M) \\
& \quad=x+e^{-r_{1}\left(x+x_{1}\right)}\left\{\frac{\alpha_{1}-r_{1}}{\alpha_{1}}\left[\frac{r_{2}}{\alpha_{1}+r_{2}} x_{1}+\frac{1}{\alpha_{1}}+\frac{\alpha_{1}}{\alpha_{1}+r_{2}} D_{1}\right]-D_{1}\right\} .
\end{aligned}
$$

To show that the second summand in the r.h.s. of the previous formula vanishes, we compute

$$
\begin{aligned}
& \frac{\alpha_{1}-r_{1}}{\alpha_{1}}\left[\frac{r_{2}}{\alpha_{1}+r_{2}} x_{1}+\frac{1}{\alpha_{1}}+\frac{\alpha_{1}}{\alpha_{1}+r_{2}} D_{1}\right]-D_{1} \\
&=x_{1} \frac{\alpha_{1}-r_{1}}{\alpha_{1}} \frac{r_{2}}{\alpha_{1}+r_{2}}+\frac{\alpha_{1}-r_{1}}{\alpha_{1}^{2}}+\frac{\alpha_{1}-r_{1}}{\alpha_{1}+r_{2}} D_{1}-D_{1} \\
&=x_{1} \frac{r_{2}}{\alpha_{1}} \frac{\alpha_{1}-r_{1}}{\alpha_{1}+r_{2}}+\frac{\alpha_{1}-r_{1}}{\alpha_{1}^{2}}-\frac{r_{1}+r_{2}}{\alpha_{1}+r_{2}} D_{1} .
\end{aligned}
$$

Taking into account (4.14) and the first equation in (4.15), we obtain

$$
x_{1}=\frac{D_{1} F_{1}-E_{1}}{F_{1}-1}=\frac{r_{1}}{r_{2}} \frac{\alpha_{1}+r_{2}}{\alpha_{1}-r_{1}}\left(D_{1} F_{1}-E_{1}\right),
$$

and substitute

$$
\begin{aligned}
x_{1} \frac{r_{2}}{\alpha_{1}} \frac{\alpha_{1}-r_{1}}{\alpha_{1}+r_{2}}+\frac{\alpha_{1}-r_{1}}{\alpha_{1}^{2}} & -\frac{r_{1}+r_{2}}{\alpha_{1}+r_{2}} D_{1} \\
=\frac{r_{1}}{\alpha_{1}}\left(D_{1} F_{1}-\right. & \left.E_{1}\right)+\frac{\alpha_{1}-r_{1}}{\alpha_{1}^{2}}-\frac{r_{1}+r_{2}}{\alpha_{1}+r_{2}} D_{1} \\
& =D_{1}\left(\frac{r_{1}}{\alpha_{1}} F_{1}-\frac{r_{1}+r_{2}}{\alpha_{1}+r_{2}}\right)-\frac{r_{1}}{\alpha_{1}} E_{1}+\frac{\alpha_{1}-r_{1}}{\alpha_{1}^{2}}=0,
\end{aligned}
$$

in view of the definitions of $E_{1}$ in (4.2) and $F_{1}$ in (4.3). Similarly, for $x<-x_{1}$, we obtain $\mathbf{E}_{x} Q_{1}(I)+Q_{2}(M)=-x$. This concludes the verification of (2.3). 
We now verify (2.5). From the computations above, we obtained that

$$
D_{1}=\frac{\alpha_{1}-r_{1}}{\alpha_{1}}\left[\frac{r_{2}}{\alpha_{1}+r_{2}} x_{1}+\frac{1}{\alpha_{1}}+\frac{\alpha_{1}}{\alpha_{1}+r_{2}} D_{1}\right]
$$

This gives that, for $x \geq-x_{1}$, we have:

$$
\mathbf{E}_{x} Q_{1}(I)=D_{1} e^{-r_{1}\left(x+x_{1}\right)}
$$

Applying the corresponding same arguments (or considering the the dual Lévy process $\left.\left\{-X_{t}: t \geq 0\right\}\right)$, for $x<x_{2}$ we obtain

$$
\mathbf{E}_{x} Q_{2}(M)=D_{2} e^{r_{2}\left(x-x_{2}\right)} .
$$

In particular, this gives that $D_{1}>0$, and, for $-x_{1} \leq x \leq x_{2}$,

$$
\mathbf{E}_{x} Q_{1}(I)+\mathbf{E}_{x} Q_{2}(M)=D_{1} e^{-r_{1}\left(x+x_{1}\right)}+D_{2} e^{r_{2}\left(x-x_{2}\right)},
$$

so the definition (2.4) gives (4.9). To conclude with the proof, it remains to verify condition (2.5) for $x \in\left[-x_{1}, x_{2}\right]$. We take $0 \leq x \leq x_{2}$. We have

$$
\begin{aligned}
\mathbf{E}_{x} Q_{2}(M) & =\mathbf{E}\left(x+M-E_{2}-F_{2} D_{1} e^{-r_{1}\left(x+M+x_{1}\right)}\right) \\
& \geq \mathbf{E}\left(x+M-E_{2}-F_{2} D_{1} e^{-r_{1}\left(x+M+x_{1}\right)}\right) \\
& =x+\mathbf{E} M-E_{2}-D_{1} F_{2} \mathbf{E}\left(e^{-r_{1} M}\right) e^{-r_{1}\left(x+x_{1}\right)} \\
& =x-D_{1} e^{-r_{1}\left(x+x_{1}\right)}=x-\mathbf{E}_{x} Q_{1}(I),
\end{aligned}
$$

in view of the definition of $E_{2}$ in (4.2), $F_{2}$ in (4.3), and formulas (4.16) and (4.17). For $-x_{1} \leq x \leq 0$ the symmetric computation completes the verification of (2.5). This completes the verification of all the hypothesis of Theorem 2.1, and the proof of Theorem 4.1 .

Remark 4.4. It is interesting to note that the function $x-E_{2}$ appearing in the first two summands in the r.h.s. of (4.12), in the terminology of Surya [15], is the averaging function of the one sided problem with payoff function $g_{2}(x)=x^{+}$(see Mordecki [11]). So the remaining term in the r.h.s. in (4.12) is a correction due to the presence of the infimum in the two-sided problem. As $x_{2}$ is the root of $Q_{2}$, we obtain $x_{2} \geq E_{2}$.

Remark 4.5. The proof of Theorem 4.1 also provides bounds to find $u$ numerically. First observe that $Q_{2}(x) \leq x$ for $x \geq 0$, so $\mathbf{E} Q_{2}(M) \leq E_{2}$. Furthermore, in view of (4.14) and (4.18), we have

$$
x_{1}=E_{1}+F_{1} D_{2} e^{-r_{2}\left(x_{1}+x_{2}\right)}=E_{1}+F_{1} \mathbf{E} Q_{2}(M) e^{-r_{2} x_{1}} \leq E_{1}+F_{1} E_{2} .
$$

In view of the previous remark, the conclusion is that

$$
E_{1}+E_{2} \leq u \leq E_{1}\left(1+F_{2}\right)+E_{2}\left(1+F_{1}\right)
$$

\subsection{Numerical examples}

To illustrate our results we consider two examples. In the first one we choose $\left(\alpha_{1}, \lambda_{1}, \alpha_{2}, \lambda_{2}, r\right)=(1,3,3,1,1)$. The thresholds are $x_{1}=1.17$ and $x_{2}=0.87$. The second example is symmetric, with $\left(\alpha_{1}, \lambda_{1}, \alpha_{2}, \lambda_{2}, r\right)=(1,1,1,1,1)$ The critical thresholds are $x_{1}=x_{2}=1.04$. The corresponding value functions (4.9) are shown in Figure 1. 

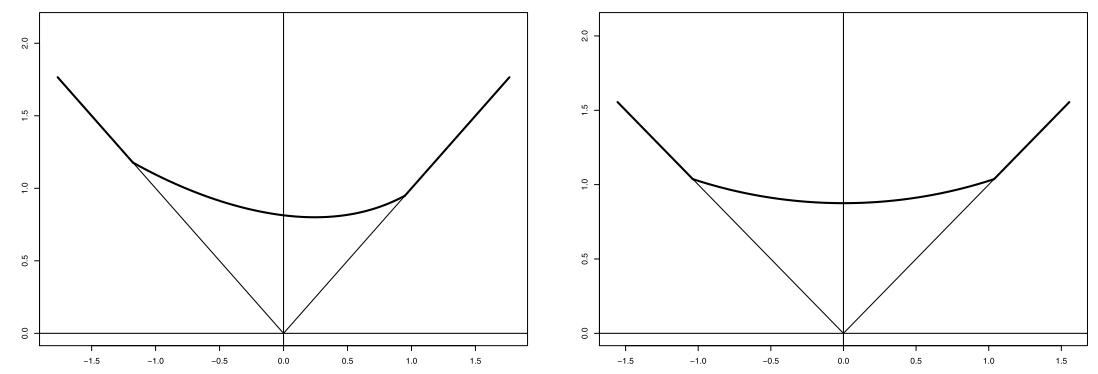

Figure 1: The payoff functions $V$ for the set of parameters $(1,3,3,1,1)$ (left) and $(1,1,1,1,1)$ (right).

\section{References}

[1] Alili, L. and Kyprianou, A.E., Some remarks on first passage of Lévy processes, the American put and pasting principles, Annals of Applied Probability, 2005, 15, 3, 2062-2080. MR2152253

[2] Bertoin, J., Lévy processes, Cambridge Tracts in Mathematics. 121. Cambridge: Cambridge Univ. Press., 1996. MR-1406564

[3] Boyarchenko, S. I., Two-Point Boundary Problems and Perpetual American Strangles in Jump-Diffusion Models (2006). Available at SSRN: https://ssrn.com/abstract $=896260$ or http://dx.doi.org/10.2139/ssrn.896260.

[4] Buonaguidi, B., Muliere, P. Bayesian sequential testing for Lévy processes with diffusion and jump components, Stochastics, 88:7, (2016), 1099-1113. MR-3529862

[5] Ming-Chi Chang and Yuan-Chung Sheu. Free boundary problems and perpetual American strangles, Quantitative Finance, 13(8), (2013), 1149-1155. MR-3175893

[6] Christensen, S., Salminen, P. and Ta, Bao Quoc. Optimal stopping of strong Markov processes. Stochastic Process. Appl. 123, no. 3, (2013), 1138-1159. MR-3005017

[7] De Donno M., Palmowski Z., Tumilewicz J. Double continuation regions for American and Swing options with negative discount rate in Lévy models. Mathematical Finance (2019), 1-32. MR-4067074

[8] Kyprianou, A. E., Introductory lectures on fluctuations of Lévy processes with applications. Springer, (2006). MR-2250061

[9] Lewis, A., Mordecki, E. Wiener-Hopf factorization for Lévy processes with negative jumps with rational transforms. Journal of Applied Probability, Vol 45, Nr. 1. (2008), 118-134. MR-2409315

[10] Mordecki, E., Optimal stopping and perpetual options for Lévy processes., Finance Stoch., 6(4) 473-493, (2002). MR-1932381

[11] Mordecki, E. Perpetual options for Lévy processes in the Bachelier model. Proceedings of the Steklov Mathematical Institute, 237, 256-264, (2002). MR-1976521

[12] Mordecki, E., and Mishura, Yu., Optimal stopping for Lévy processes with one-sided solutions, SIAM Journal on Control and Optimization, 54(5), (2016), 2553-2567. MR-3551206

[13] Mordecki, E. and Salminen, P. Optimal stopping of Hunt and Lévy processes. Stochastics 79, no. 3-4, (2007), 233-251. MR-2308074

[14] Shiryaev, A. N. Optimal stopping rules. Stochastic Modelling and Applied Probability, 8. Springer-Verlag, Berlin, 2008. MR-2374974

[15] Surya, B. A., An approach for solving perpetual optimal stopping problems driven by Lévy processes, Stochastics. An International Journal of Probability and Stochastic Processes, 79, 3-4, 337-361, (2007). MR-2308080 